\title{
MANAGEMENT OF ARTERIAL COMPLICATIONS AFTER LIVING DONOR LIVER TRANSPLANTATION
}

\author{
Khaled Zaky Mansour, Mohamed Mohamed Bahaa El-Din, Ahmed Hussein \\ Abdelhafez, Kamal Mamdouh Kamal and Mohamed Ahmed Fathi El Bahnasy
}

Department of General
Surgery, Faculty of Medicine -
Ain Shams University
Corresponds:
Mohamed Ahmed Fathi El
Bahnassy
E.male:
Bahnasy2017@yahoo.com
Tel.: 01091732303
Received: 10/3/2019
Accepted: 3/4/2019

\begin{abstract}
Background: Liver transplantation is perceived as the only curative treatment for patients with end-stage liver disease. Arterial complications are one of the most serious complications after living donor liver transplantation (LDLT).
\end{abstract}

Aim of the work: To assess the different modalities of management and outcome of early and late arterial complications and to analyze the risk factors of such complications after living donor liver transplantaiton.

Patients and Methods: This is a retrospective study in which 293 LDLT operations were done between 2008 and May 2017 at Ain Shams center for organ transplantation (ASCOT). After approval of Ethics committee and the research council, Ain shams university school of medicine, we did this retrospective cohort study that analyzed the incidence, risk factors, management and outcome of HA complications in adults and pediatrics recipients in the period from 2008 to May 2017, where patients were observed from POD 1 until the end 2017 or until death of patients.

Results: In our study early arterial complications are more higher and more serious than late arterial complications. Mortality rate in patients with early complications exceeds 50\%.In late complications it was $20 \%$.

Conclusion: Urgent surgical management is life saving in cases of early hepatic artery thrombosis (HAT). Arterial reconstruction is technically difficult. LD retransplant has been performed in a very small number worldwide. Emergency living donor retransplantation is life saving in cases of early (HAT). Arterial reconstruction using left gastric artery and splenic artery is feasible in $L D$ retransplant and arterial reconstruction after HAT. Early diagnosis and surgical or radiological intervention is the corner stone to save the recipient.

Keywords: Living donor liver transplantation, arterial complications, hepatic artery thrombosis.

\section{INTRODUCTION:}

Liver transplantation is perceived as the only curative treatment for patients with end-stage liver disease. Approximately 20$25 \%$ of patients with liver failure die while waiting for a liver transplant, and another $20-30 \%$ of patients with hepatocellular carcinoma drop off the waiting list because of tumor progression ${ }^{(1)}$.

Living donor liver transplantation (LDLT) has emerged as the alternative lifesaving treatment to deceased donor liver transplantation (DDLT). Over the past 2 decades, the number of LDLTs has steadily 


\section{Khaled Zaky Mansour, et al.,}

increased in many transplant centers, especially in $\mathrm{Asia}^{(2)}$.

Living donor liver transplantation has the following advantages over DDLT: a shorter wait time, a shorter cold ischemic time, and a better organization of the surgery time. However, donor risks are inevitable and are an undeniable problem that troubles transplant surgeons. Moreover, LDLT has a smaller biliary and vascular caliber and an additional transection step, which may potentially increase the surgical risk and the incidence of postoperative complications ${ }^{(3)}$.

As surgical techniques and postoperative managements continue to advance, the outcomes of LDLT have continued to improve $^{(4)}$.

Many complications have been reported in the recipient such as biliary complications, vascular complications, intraabdominal bleeding, intra-abdominal abscesses, ileus, bowel obstruction, pulmonary complications, deep venous thrombosis, wound dehiscence, incisional hernia, infections (bacterial, viral and fungal), ascitis, acute or chronic rejection, recurrence of previous infection with $\mathrm{HBV}, \mathrm{HCV}$ OR malignancy as $\mathrm{HCC}^{(5)}$.

Vascular complications are common cause of morbidity of liver transplantation, especially hepatic artery problems. It was reported that the hepatic artery complication rate to be approximately $5 \%-16 \%{ }^{(6)}$.

Living donor liver transplantation patients may suffer from a higher incidence of arterial complications due to the smaller vessel diameter, the insufficient length for reconstruction and the greater risk of a twist of the vascular pedicle. These complications include mainly hepatic artery thrombosis and hepatic artery stenosis ${ }^{(6)}$.

Doppler ultrasonography has high sensitivity for the diagnosis of hepatic artery thrombosis by performing serial examinations at frequent intervals during the first 1 to 2 weeks post-transplant. Hepatic artery thrombosis can be detected before it becomes clinically obvious ${ }^{(7)}$.

Early diagnosis permits immediate thrombectomy and revascularization before the patient deteriorates. If there is suspicion for hepatic artery thrombosis, one can choose to delineate the anatomy with angiography or proceed urgent re-exploration. Angiography offers non-operative method to diagnose and potentially treat with balloon angioplasty ${ }^{(7)}$.

If there is a delay in the diagnosis, or if ultrasound is questionable, selective arteriography may be employed to diagnose the site of thrombosis and to begin therapeutic thrombolysis. Decisions on retransplantation are made based on the clinical condition, patency of the vessels, and appearance of late complications as biliary stricture ${ }^{(7)}$.

Late hepatic artery thrombosis (HAT) is a rare complication after liver transplantation conventionally described as occurring more than 30 days after surgery ${ }^{(8)}$.

Late hepatic artery thrombosis often asymptomatic because of the development of a rich collateral network. Attempts at operative revision should not be undertaken, as a large majority survives with normal allograft function, and any operative procedure carries the risk of destroying the graft-sustaining collaterals. Significant late allograft dysfunction needs careful monitoring for septic complications, biloma and cholangitis. Attempts at graft salvage in this population are universally unsuccessful ${ }^{(9)}$.

Hepatic artery stenosis, although usually asymptomatic, will eventually progress to hepatic artery thrombosis. Frequently, patients develop biliary strictures and bile leaks. Hepatic artery stenosis may be detected on surveillance Doppler. Dampened waveforms with decreased resistivelindices (RI) and slow peak velocities suggest hepatic artery stenosis. Stenosis should be suspected when the RI is $<0.5$ or the systolic 
ascending time (SAT) is $>10 \mathrm{msec}$. The diagnosis should be confirmed by angiography. If diagnosed in the immediate postoperative period, planned exploration and revision of the arterial anastomosis should be undertaken. Although conventional treatment is either surgical repair or a re-transplant, percutaneous transluminal angioplasty (PTA) or stent placement is becoming predominant ${ }^{(10)}$.

\section{AIM OF THE WORK:}

This retrospective study aims to assess the different modalities of management and outcome of early and late arterial complications and to analyze the risk factors of such complications after living donor liver transplantaion.

\section{PATIENTS AND METHODS}

This is a retrospective study in which 293 LDLT operations were done between 2008 and May 2017 at Ain Shams center for organ transplantation (ASCOT).

After approval of Ethics committee and the research council, Ain shams university school of medicine, we did this retrospective cohort study that analyzed the incidence, risk factors, management and outcome of HA complications in adults and pediatrics recipients in the period from 2008 to May 2017, where patients were observed from POD 1 until the end 2017 or until death of patients.

The data were collected from our records in the LT unit and written informed consents were obtained from both donors and recipients regarding operations and researches.

Donor criteria: Age: >21years old except in offspring and siblings $>18$. Compatible blood group. BMI $<28$. No comorbidities. No previous history of major abdominal operation.
Exclusion criteria: only one patient excluded from the study as he died intraoperative.

\section{Study Procedures:}

Preoperative parameters: Recipient age, gender, primary disease, Child Pugh and MELD score, co-morbidity (DM, HTN,...), and previous vascular thromboses (HA, PV and HV).

\section{All donors and recipients passed into the following preoperative preparation:}

Preoperative workup: Clinical evaluation and following labs, ALT, AST, blood group, Rh type, HCV Ab, HBV sAg, HIV $\mathrm{Ab}$ and $\mathrm{HBcAb}$ IgG. CBC with differential count, ESR, CRP, liver functions (total bilirubin, direct bilirubin, albumin, total protein, ALP, GGT, amylase), renal profile (BUN, creatinine, uric acid, Na, K), lipid profile (cholesterol, HDL, LDL and Triglycerides), bleeding profile (PT, PTT, INR, Prothrombin concentration, protein $C$, protein $\mathrm{S}$, factor $\mathrm{V}$ concentration, antithrombin III), serum ferritin, Fasting and 2 hours postprandial blood sugar, Chloride, Calcium, Glucose, Phosphorus, viral markers (HAV Ab IgM, HAV Ab IgG, $\mathrm{HBsAb}$ titre, HBeAb, HBeAg, HBc IgM), Urine for drug abuse for (Cocaine, Cannabis, Barbiturates, Benzodiazipines, Opiates, Amphetamines), complete urine analysis, complete stool analysis, Bilharizaial $\mathrm{Ab}$ titre, factor $\mathrm{V}$ leiden gene mutation, CRP quantitative. Viral markers (HBV PCR qualitative, $\mathrm{HCV}$ PCR qualitative, EBV lgM, EBV IgG, CMV IgG, CMV IgM, HSV type $1 \mathrm{IgM}$, HSV type $1 \mathrm{IgG})$, coagulation profile (protein $\mathrm{C}$, protein $\mathrm{S}$, antithrombin III, lupus anticoagulant, anticardiolipin $\mathrm{Ab}$ $\mathrm{IgM}$, anticardiolipin $\mathrm{Ab}$ Ig $\mathrm{G}$ ), tumor markers (CEA, CA 19-9, PSA, CA125, CA 15-3, $\alpha$-FP), circulating Bilharizaial Ag if needed.

Pre-operative imaging procedures: Abdominal duplex ultrasonography. Spiral CT. Abdomen for exclusion of any 
unrecognized diseases (for both donors and receipients). CT. Arteriography, portography, venography to assess arterial and venous anatomy. This is done mainly in the donor to precisely predict how many hepatic arterial stumps there will be on a graft. CT. Volumetry was done in the donor to estimate the volume of the whole liver, right liver volume (to be compared to the weight of the recipient to get a satisfactosry GRWR), the remaining liver volume (which is called the residual liver volume and it is one of the most important factors that affects the donor morbidity and should be more than $35 \%$ of the total liver volume). MRCP was done to delineate the biliary anatomy and was compared with intraoperative cholangiography. Liver biopsy was routinely done in all donors for evaluation.

Intraoperative parameters Duration of the operation per hours, actual graft weight, actual graft recipient weight ratio (GRWR), number of arterial reconstructions, cold and worm ischemia times per minute, blood and plasma transfusion per unit.

Donor operation: The donor operation was performed through a right subcostal incision extended to the upper midline under general anesthesia. Intraoperative cholangiography was used to define the biliary anatomy of donors, the right or left lobes of the liver were mobilized and the vena cava was dissected. The type of liver graft used was dependent on the body build of the recipient and on the calculated segmental volume of the donor liver. The CUSA device was used to divide the liver parenchyma without inflow occlusion. The falciform ligament was reconstructed, the stumps of the divided hepatic and portal veins were closed by continuous non-absorbable sutures, after graft harvesting, it was perfused in the back table with Hydroxy tryptophan ketoglutarate (HTK) solution and weighted to determine the actual GRWR.

Recipient surgery: A (J shaped) hockey stick incision including a small upper midline incision and a right subcostal incision was used to enter the abdomen. Following careful laparotomy, the round ligament of the liver is tied and cut. Double retractors on the right and left costal arch provide excellent exposure. Mobilization of the liver with piggyback, When dissecting around the IVC to the cranial side, the IVC ligament appears in the upper third of the right margin of the IVC. Dissection of the IVC ligament is started at the cranial end of the ligament and some space is made between the ligament and the right hepatic vein. Hilum dissection with section of right and left branches of HA, then dissection and section of bile duct, so total hepatectomy is done with preservation of the RHV and common trunk of MHV and LHV. The ready graft for transplantation is brought out to the recipient and we start with RHV anastmosis to the RHV stump in the IVC with prolene 4/0 and the inferior right hepatic vein (Makuuchi) is anastmosed to the IVC directly using prolene $5 / 0$ if preserved. Then anastmosis of the portal vein is done using prolene $6 / 0$ and at this step venous declamping is done where the graft is flushed with the portal blood.

The HA anastomosis was performed using 4 to $4.5 \mathrm{x}$ loupe magnification with interrupted 8/0 monofilament Prolene which facilitates secure sutures with good intima adaptation. Hepatic artery reconstruction was performed by end to end anastomosis after reconstructing of both hepatic and portal veins followed by the reperfusion of the graft using Donor right HA to recipient right HA in case of right lobe graft and using donor left HA to recipient left HA in case of left lobe graft. Splenic artery was anastomosed to RHA in four cases due to inadequate flow in $\mathrm{HA}$ as a result of atherosclerosis, intimal dissection and repeated pretransplant TACE.

The HA inflow was considered inadequate if no arterial jetting can be obtained despite adequate dissection down till common HA with ligation of the gastro duodenal artery and heparin flushing before arterial 
anastomosis or abnormal Doppler finding after it. If this occurred, the splenic artery would be used for arterial anastomosis.

The SA was exposed by reflecting downward the stomach and incising the retroperitoneum at the upper edge of the pancreas. Usually in the cirrhotic patient with splenomegaly, the SA is dilated, tortuous, and can be easily encircled. After the revascularization of the graft through the portal vein, the SA was clamped and the distal end is ligated and divided as far as possible. The proximal end was turned to the right and an end-to-end anastomosis was performed with the HA of the donor using $8 / 0$ prolene interrupted stitches, to be wider on the SA side to overcome discrepancy in the arterial diameter between SA and HA.

Lt gastric artery was anastomosed to Rt $\mathrm{HA}$ in 2 patients.

Rt HA was anastomosed to Lt HA (single anastomosis) in 5 patients.

In grafts with two hepatic arterial stumps; the more sizable artery of the graft will be anastomosed to recipient Rt HA and if there is backflow there is no need to reconstruct the second graft artery. Double anastomosis was done in 5 cases due to inadequate backflow in the second graft artery.

Doppler ultrasonography is done to assess the vascularity of the graft after the anastmoses of the hepatic veins, the portal vein and hepatic artery.

Then anastmosis of the bile duct is done using 6/0 PDS with or without stent applied and at this step intraoperative cholangiography is done to assess the patency of the biliary tree.

Finally abdominal wall closure is done in layers anatomically after application of intrabdominal drains in the determined sites (morrison pouch, hepatic pedicle and left subdiaphragmatic space).
Postoperative management: Based on our policy

Immunosuppression protocols: the standard is combined 3drugs: calcineurin inhibitors (CNIs), steroids and mycophenolate mofetil (MMF). Cyclosporine (CsA) was used when neurotoxicity or nephrotoxicity developed with Tacrolimus. When CNIs are contraindicated or their side effects halt their use, sirolimus (SRL) was given at an initial dose of $3 \mathrm{mg} / \mathrm{m} 2$ and adjusted over time to achieve blood trough levels of approximately $5 \mathrm{e} 8 \mathrm{ng} / \mathrm{mL}$.

Routine anticoagulant and antiplatelet therapy: antiplatelet agents were started intraoperative and started post operative when platelets $>50.000$ and continued for life. LMW heparin was started according to the drains and continued for one month.

Doppler ultrasonography: All patients underwent Doppler ultrasonography every day during the first week postoperative, every 48 hours during the second postoperative week, twice per week till 1 month post operative and then once per week till 3 months to confirm patency. Also Doppler is performed urgently in case of marked elevation of the serum bilirubin and transaminase levels.

Diagnosis of HA complications: was suspected when the LFT results became abnormal or when doppler ultrasound revealed poor or no blood flow within the hepatic vessels. The complications were confirmed on either computerized tomogramphy angiography (CTA), magnetic resonance angiography (MRA), or formal conventional angiography as necessary. HA complications were defined as early complications when occurring within the first 30 days of LT and as late complications if diagnosed after 30 days of LT.

Treatment of HA complications: Prompt surgical thrombectomy and reconstruction were always our first choice 
in early cases while angiographic percutaneous thrombectomy and thrombolysis were used in late cases. However, medical treatment was the choice in some cases.

\section{Statistical analysis}

All data were tabulated and processed with SPSS software (Statistical Product and Service Solutions, version 21, SSPS Inc, Chicago, IL, USA) and Windows XP (Microsoft Corporation, Redmond,
Washington, USA). Qualitative data were expressed in frequency and percentage and analyzed with the chi-square or Fisher exact tests. Quantitative data were expressed as the mean and standard deviation and were compared with the $\mathrm{t}$ or Mann whitney $\mathrm{U}$ tests. Comparison between patients with and without HA complications was done using Univariate analysis. The Kaplane Meier method was applied for survival analysis and compared using log-rank tests. In all tests, a $\mathrm{P}$ value of $<0.05$ was considered significant.

\section{RESULTS}

Table (1): Types of HA reconstruction:

\begin{tabular}{|l|c|c|}
\hline & No. & $\%$ \\
\hline Single & 287 & $98.3 \%$ \\
\hline Multiple(double) & 5 & $1.7 \%$ \\
\hline Rt HA to Rt HA & 271 & $92.8 \%$ \\
\hline Lt HA toLt HA & 6 & $2 \%$ \\
\hline Rt HA to Lt HA & 4 & $1.4 \%$ \\
\hline Rt HA to splenic & 4 & $1.4 \%$ \\
\hline Rt HA to lt gastric.a & 2 & $0.7 \%$ \\
\hline
\end{tabular}

The mean actual graft weight and GRWR were $759.8 \pm 239.04 \mathrm{~g}$ and $1.2 \pm 0.57$ respectively. The mean cold and warm ischemia times were $69.9 \pm 46.4 \mathrm{~min}$ and $49.9 \pm 15.6 \mathrm{~min}$ respectively. The mean intraoperative blood and plasma transfusions were $6.03 \pm 7$ and $7 \pm 8.3$ units respectively (Table 3 ).

Table (2): Characteristics of patients.

\begin{tabular}{|l|c|}
\hline Recipient age (years) (Mean \pm SD) & $43.9 \pm 17.1$ \\
\hline Recipient gender & $249(85.3 \%)$ \\
\hline Males & $43(14.7 \%)$ \\
\hline Females & $26(8.9 \%)$ \\
\hline Child class & $92(31.5 \%)$ \\
\hline A & $174(59.6 \%)$ \\
\hline B & $16 \pm 4.2$ \\
\hline C & $91(31 \%)$ \\
\hline MELD score (Mean \pm SD) & $37(12.7 \%)$ \\
\hline Co morbidity & $286(98 \%)$ \\
\hline Preoperative PVT & $6(2 \%)$ \\
\hline Graft type & $287(98.3 \%)$ \\
\hline Right lobe & $5(1.7 \%)$ \\
\hline Left lobe & $759.8 \pm 239.04$ \\
\hline HA anastomosis & $1.2 \pm 0.57$ \\
\hline Single & $20(9.4 \%)$ \\
\hline Multiple & $69.9 \pm 46.4$ \\
\hline Actual graft weight (Mean \pm SD) &
\end{tabular}




\begin{tabular}{|l|c|}
\hline Warm ischemia time $(\mathrm{min})(\mathrm{Mean} \pm \mathrm{SD})$ & $49.9 \pm 15.6$ \\
\hline Intraoperative blood transfusion (units) & $6.03 \pm 7$ \\
\hline Intraoperative plasma transfusion (units) & $7 \pm 8.3$ \\
\hline Duration of operation (hours) (Mean \pm SD) & $12.5 \pm 3.1$ \\
\hline Hospital stay (post operative) (days) (Mean \pm SD) & $23.8 \pm 16.6$ \\
\hline
\end{tabular}

Table (3): Indications of LT in adults.

\begin{tabular}{|l|c|}
\hline HCV & $164 / 292(56.2 \%)$ \\
\hline HCV cirrhosis + HCC & $90(30.8 \%)$ \\
\hline HBV cirrhosis+ HCC & $7(2.4 \%)$ \\
\hline Cryptogenic cirrhosis & $10(3.4 \%)$ \\
\hline HBV & $6(3.2 \%)$ \\
\hline BCS & $2(0.7 \%)$ \\
\hline PSC & $2(0.7 \%)$ \\
\hline PBC & $1(0.4 \%)$ \\
\hline Autoimmune hepatitis & $5(1.7 \%)$ \\
\hline Alcoholic cirrhosis & $1(0.4 \%)$ \\
\hline Biliary atresia & $3(1 \%)$ \\
\hline Retransplantation & $1(0.4 \%)$ \\
\hline
\end{tabular}

HCV: Hepatitis C virus, HCC: Hepatocellular carcinoma, HBV: Hepatitis B virus, BCS: Budd chiari syndrome, PSC: primary sclerosing cholangitis, PBC: Primary biliary cirrhosis.

Primary disease: The most frequent indications were HCV 164/292. (56.2\%) followed by HCC 97/292 (33.2\%).

\section{Predictors of HA complications:} There was a trend towards statistical significant higher complications with male recipients, there was no significant correlation between cold ischemia time,
GRWR, preoperative PVT, previous TACE or amount of blood transfusion and incidence of arterial complications in our work. Also there is no significant correlation between age of the recipient, presence of comorbidities, operative time, and number of arterial anastomosis and incidence of arterial complications.

Table (4): Predictors of HA complications.

\begin{tabular}{|c|c|c|}
\hline Category & HA complications number (\%) & p-value \\
\hline Number of patients & \multicolumn{2}{|c|}{$16 / 292(5.5 \%)$} \\
\hline \multicolumn{3}{|c|}{ Recipient age $>18$ years } \\
\hline Yes & $16 / 289(5.5 \%)$ & $>0.05$ \\
\hline No & $0 / 3(0 \%)$ & \\
\hline \multicolumn{3}{|l|}{ Recipient gender } \\
\hline Males & $15 / 239(6.3 \%)$ & 0.1 \\
\hline Females & \multicolumn{2}{|c|}{$1 / 43(2.3 \%)$} \\
\hline \multicolumn{3}{|l|}{ Co-morbidity } \\
\hline Yes & $6 / 91(6.6 \%)$ & $>0.05$ \\
\hline No & \multicolumn{2}{|c|}{$10 / 201(5 \%)$} \\
\hline \multicolumn{3}{|l|}{ Preoperative PVT } \\
\hline Yes & $2 / 37(5.4 \%)$ & 0.049 \\
\hline No & \multicolumn{2}{|c|}{$14 / 252(5.6 \%)$} \\
\hline \multicolumn{3}{|l|}{ Graft type } \\
\hline Right lobe & $16 / 286(5.6 \%)$ & $>0.05$ \\
\hline Left lobe & \multicolumn{2}{|l|}{$0 / 6(0 \%)$} \\
\hline HA anastomosis & & \\
\hline
\end{tabular}


Khaled Zaky Mansour, et al.,

\begin{tabular}{|l|c|c|}
\hline Single & $16 / 287(5.6 \%)$ & $>0.05$ \\
\hline Multiple & \multicolumn{2}{|c|}{$0 / 5(0 \%)$} \\
\hline TACE & $2 / 39(5.1 \%)$ & $>0.05$ \\
\hline Yes & \multicolumn{2}{|c|}{$14 / 253(5.5 \%)$} \\
\hline No & \multicolumn{2}{|c|}{} \\
\hline
\end{tabular}

Table (5): Predictors of HA complications.

\begin{tabular}{|c|c|c|c|}
\hline Category & $\begin{array}{c}\text { HAC } \\
\text { (Mean } \pm \text { Std. } \\
\text { deviation) }\end{array}$ & $\begin{array}{c}\text { No HAC } \\
\text { (Mean } \pm \text { Std. } \\
\text { deviation) }\end{array}$ & p-value \\
\hline MELD & $14.9 \pm 4.3$ & $15.7 \pm 4.2$ & $>0.05$ \\
\hline Actual graft wt & $831.2 \pm 231.3$ & $774.2 \pm 211.4$ & 0.08 \\
\hline Actual GRWR & $1.2 \pm 0.4$ & $1.2 \pm 0.6$ & $>0.05$ \\
\hline Cold ischemia time/minutes & $63.9 \pm 35.1$ & $70.1 \pm 48.1$ & $>0.05$ \\
\hline Warm ischemia time/minutes & $45.8 \pm 17.2$ & $50 \pm 15.7$ & $>0.05$ \\
\hline Blood transfusion (units) & $6.4 \pm 6.1$ & $5.9 \pm 7$ & $>0.05$ \\
\hline Plasma transfusion (units) & $7.1 \pm 9.3$ & $6.7 \pm 7.8$ & $>0.05$ \\
\hline Operative time/h & $12.4 \pm 2.1$ & $12.5 \pm 2.9$ & $>0.05$ \\
\hline
\end{tabular}

HA complications and their hallucinations on 6th day, elevated liver management: The incidence of HA problems was $16(5.5 \%)$, in the form of 11 $(3.8 \%)$ early HA complications (the $1 \mathrm{st}$ month post operative) and $5(1.7 \%)$ late HA complications. Early HA complications include: nine patients with HAT, one patient with bleeding and one patient with pseudoaneurysm. Early HAT occurred in 9 patients (3.1\%), two patients developed HAT at day 14-16 post operative diagnosed with Doppler US, elevated liver enzymes and confirmed by $\mathrm{CT}$ angiography and they died. Early HAT occurred in sex patients on1st or2nd day post operative diagnosed with clinical picture, marked elevation of liver enzymes and Doppler US. They are treated surgically by removing the thrombus and repeated HA anastomosis except in one of them arterial reconstruction was done using it gastric artery. Three patients improved and discharged. HAT occurred again in two patients after 8-10 days and they died. The last patient died due to pneumonia. One patient developed enzymes. Doppler US and CT angiography confirmed the diagnosis of HAT and he was successfully urgently retransplanted within 48 hours using left gastric artery. In the retransplant, we used a right lobe graft with a $10 \%$ steatosis and graft recipient weight ratio (GRWR) 0.84 with no variants in the hepatic veins, arteries, biliary and portal anatomy. One patient developed bleeding from site of anastomosis on day 1post operative needed re exploration and secured by 2 stitches. One patient developed pseudoaneurysm after two weeks and he died during angiography with stenting from ruptured pseudoaneurysm. Late hepatic artery thrombosis occurred also in 4 patients $(1.4 \%)$ diagnosed with Doppler and confirmed with C-T angiography; 2 hepatic arteries recanalized with anticoagulant after 5 and 7 months respectively. The 3 rd patient developed recurrent cholangitis and intra hepatic biliary abscess at segment VIII which was successfully managed surgically by liver resection (Figure 2). 

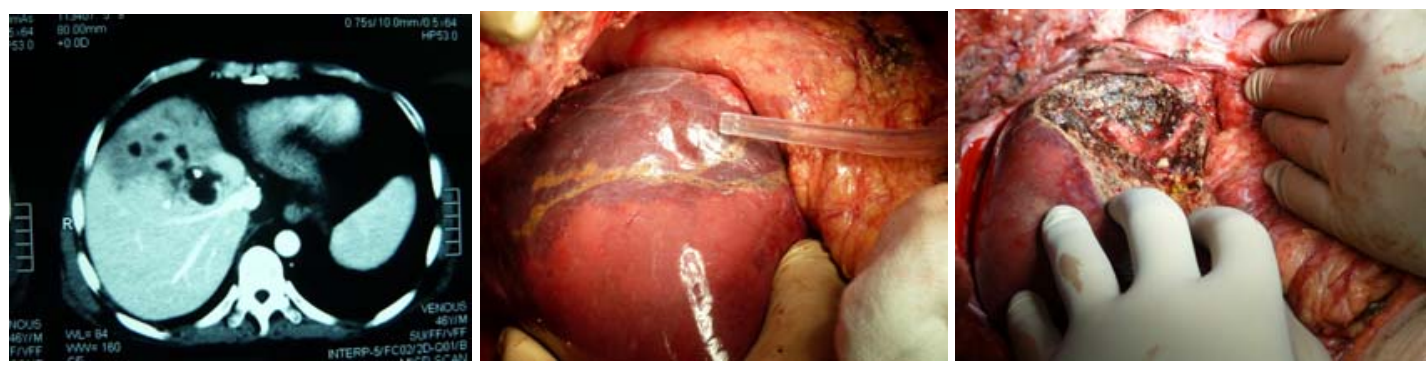

Figure (1): A) Abdominal CT showing Intra-hepatic (graft) biliary abscess. B) Intra operative intra hepatic abscess before and C) after resection segment VIII.

The last patient developed multiple intra hepatic abscesses and he died while waiting for retransplantation. Hepatic artery stenosis occurred in 1 patient $(0.75 \%)$ after 35 days successfully treated by interventional radiology dilatation with stent insertion.

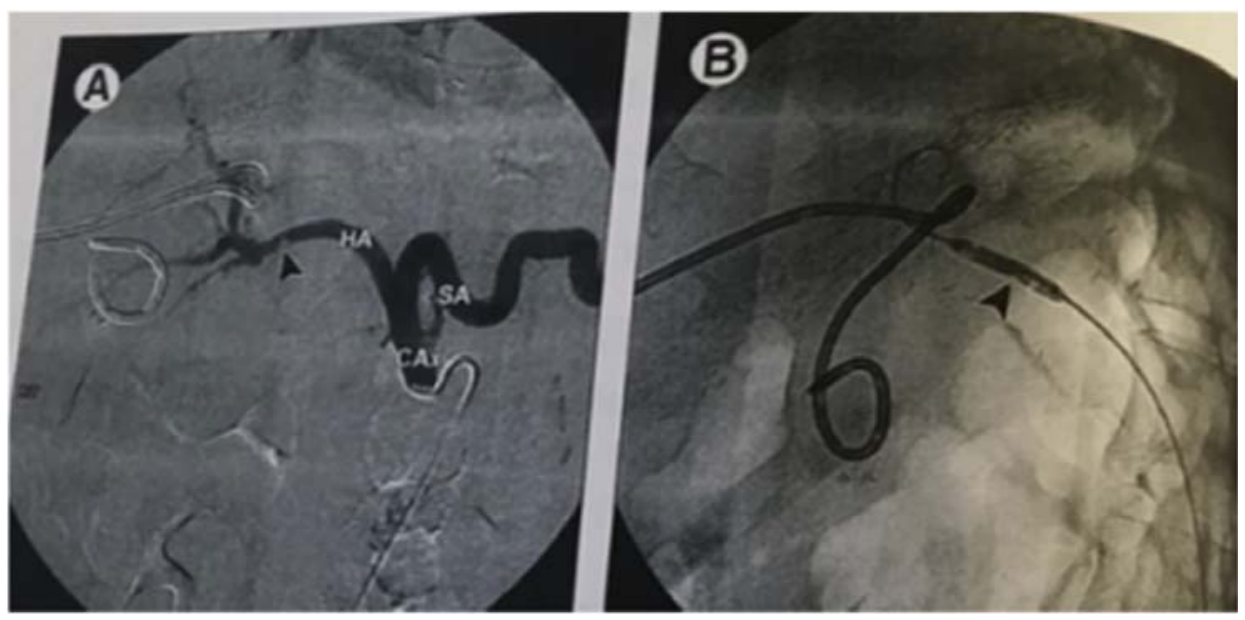

Figure (2): Patient with HAS underwent successful angiographic dilatation.

Outcome of patients: sixteen patients developed arterial complications divided into early 11 (within the first month) and 5 late complications. The mortality rate was $55 \%$ in the patients with early HA complications. The mortality rate was $20 \%$ in patients with late HA complications.

\section{DISCUSSION}

Liver transplantation has become the treatment of choice for ESLD in adults and children. Despite technical advances and improvements in postoperative care, VC after LT continues to be a major cause of mortality or graft loss. The rate of VC after LT ranges from $5 \%$ to $20 \%{ }^{(1)}$.

Similarly, the overall incidence of HA complications was $5.5 \%$ in our study.
Hepatic artery reconstruction should be performed using the most reliable procedure. A graft hepatic artery to be reconstructed in LDLT usually has a narrower caliber and a shorter stump compared to the arteries used during cadaveric liver transplantation. Microvascular surgery for hepatic artery reconstruction in LDLT decreased the number of hepatic artery complications $^{(11 \& 12)}$.

In our center (ASCOT) The HA reconstruction was performed using 4 to $4.5 \times$ loupe magnification with interrupted 8/0 monofilament Prolene by end to end anastomosis after reconstructing of both hepatic and portal veins followed by the reperfusion of the graft using Donor right $\mathrm{HA}$ to recipient right $\mathrm{HA}$ in case of right lobe graft and using donor left $\mathrm{HA}$ to 


\section{Khaled Zaky Mansour, et al.,}

recipient left HA in case of left lobe graft. Splenic artery was anastomosed to RHA in four cases due to inadequate flow in HA as a result of atherosclerosis, intimal dissection and repeated pretransplant TACE.

The HA inflow was considered inadequate if no arterial jetting can be obtained despite adequate dissection down till common HA with ligation of the gastro duodenal artery and heparin flushing before arterial anastomosis or abnormal Doppler finding after it. If this occurred, the splenic artery would be used for arterial anastomosis.

Lt gastric artery was anastomosed to Rt HA in 2 patients.

Rt HA was anastomosed to Lt HA in 5 patients.

In grafts with two hepatic arterial stumps; the more sizable artery of the graft will be anastomosed to recipient Rt HA and if there is backflow there is no need to reconstruct the second graft artery. Double anastomosis was done in 5 cases due to inadequate backflow in the second graft artery.

HAT is a serious problem; it is associated with increased morbidity, graft loss, and mortality, its incidence after LDLT varies from $3 \%$ to $10 \%$. Similarly, it was $3.1 \%$ in our study.

The treatment options for HAT include urgent revascularization, either with the native HA following thrombectomy or with HA alternatives.

Re-transplantation is the $1^{\text {st }}$ choice of treatment of early HAT but Particularly in Egypt, DDLT is not performed and, in the setting of LDLT, obtaining a second graft is quite difficult and, therefore, early detection and prompt management of HAT is crucial (13)

Early diagnosis of HAT permits immediate surgical thrombectomy and revascularization before the patient deteriorates, and ultimately may help protect patients with HAT from the need for a retransplant ${ }^{(13)}$.

The difficulty of recipient operation depends mainly on the timing of exploration and revascularisation. In our experience, early urgent exploration within the first few hours post-transplant does not entail a difficult dissection process, because the primary liver graft has few adhesions around it. Technical feasibility should be considered first. The most important concern is the availability of a hepatic arterial inflow source. It is frequently not feasible to use proper hepatic artery branches again in recipients with hepatic artery thrombosis ${ }^{(13)}$.

A reliable source, is the right gastroepiploic artery because it can be easily mobilized toward the hepatic hilum after detachment from the greater curvature of the stomach and can be promptly enlarged in case of a size mismatch after splenic artery ligation. The clinical significance of this artery has been proven in LD transplants. If it is not possible to use this artery in the retransplant, the interposition artery graft from the autogenous inferior mesenteric artery or sigmoidal artery can be used, or a fresh arterial DD graft should be procured (14)

In our center (ASCOT) we used splenic artery as an alternative to HA in four cases due to inadequate flow in HA as a result of atherosclerosis, intimal dissection and repeated pretransplant TACE.

All 4 patients are alive without any complications related to the arterial anastomosis during a follow-up period that ranged from 4- 18 month. Splenic infarction, pancreatitis or other related complications were never observed following ligation of the SA.

In the second transplant, we used the left gastric artery of the recipient for anastomosis with the right hepatic artery of the graft. In another case, we used left 
gastric artery for arterial reconstruction after arterial thrombosis for 2 times.

So splenic artery and lt gastric artery are good alternatives to HA in cases of inadequate flow in $\mathrm{HA}$ due to different causes based on our experience.

Various factors contributing to development of HA thrombosis have been proposed: multiple anastomoses, prolonged cold ischemic time, acute rejection. GRWR $<4 \%$ and massive blood transfusion (7). Conversely, there was a trend towards statistical significant higher complications with male recipients, there was no significant correlation between cold ischemia time, GRWR, preoperative PVT, previous TACE or amount of blood transfusion and incidence of arterial complications in our work. Also there is no significant correlation between age of the recipient, presence of comorbidities, operative time, and number of arterial anastomosis and incidence of arterial complications.

Late hepatic artery thrombosisis often asymptomatic because of the development of a rich collateral network. Attempts at operative revision should not be undertaken, as a large majority survives with normal allograft function, and any operative procedure carries the risk of destroying the graft-sustaining collaterals. Significant late allograft dysfunction needs careful monitoring for septic complications, biloma and cholangitis. Attempts at graft salvage in this population are universally unsuccessful ${ }^{(9)}$.

Similarly in our (ASCOT) management of late HAT was mainly non surgical. Two patients had anticoagulation therapy and one patient underwent segmental resection due to intrahepatic abscesses. Another patient died while waiting for retransplantation.

HAS ranges from $2 \%$ to $5 \%$.It was $0.3 \%$ in our study. Regarding their management, endovascular intervention was successful treatment in Steinbrück et al. (7) study.
Similarly, angiography was the main treatment option of our patients with late HAS.

Hepatic artery pseudoaneurysms (HAPsAs) are uncommon occurring in $0.4 \%$ of patients after liver transplantation. HA-PsAs are classified into intra- and extrahepatic PsAs. Their etiology, clinical course, and radiologic features differ from one another. Due to this, identifying their location (intraversus extrahepatic) is an important consideration and in turn they are discussed as 2 separate entities ${ }^{(15)}$.

In our (ASCOT) one patient developed HA pseudoaneurysm and he died during angiography and stenting due to rupture and extravasation.

Arterial pseudoaneurysms can be considered insidious, delayed, or contained arterial ruptures. They present with fevers, gastrointestinal hemorrhage (including hemobilia), sentinel and occasionally significant intra-abdominal bleeding and mass effect on the biliary tract causing obstructive jaundice ${ }^{(15)}$.

Since there are concerns that many of these extrahepatic pseudoaneurysms are actually mycotic pseudoaneurysm (postoperative arteritis), it is reasonable to approach these rare cases in a similar manner to arterial rupture, that is, wide arterial excision with regional debridement and extra-anatomical autologous vascular bypass. However, endoluminal management has also been described with successful results.

Endoluminal management includes coil embolization of the pseudoaneurysm if the neck of the pseudoaneurysm is narrow. If the neck is wide, bridging the artery across the pseudoaneurysm with a bare stent or stent graft has been described. Technical details for pseudoaneurysm embolization are similar to intrahepatic pseudoaneurysm embolization with the exception that the main hepatic artery is not dispensable and 


\section{Khaled Zaky Mansour, et al.,}

that coil embolization of the main graft hepatic artery is unacceptable unless there is active uncontrolled, life-threatening extravasation from a ruptured pseudoaneurysm. Technical details for pseudoaneurysm stenting are similar to endoluminal angioplasty or stent placement for the treatment of hepatic artery stensois $(\mathrm{HAS})^{(16)}$.

In our (ASCOT) one patient developed bleeding from site of anastomosis on day 1post operative needed re exploration and secured by 2 stitches.

\section{Conclusion:}

Urgent surgical management is life saving in cases of early HAT. Arterial reconstruction is technically difficult. LD retransplant has been performed in a very small number worldwide. Emergency LR is life saving in cases of HAT. Arterial reconstruction using left gastric artery is feasible in LD retransplant and arterial reconstruction after HAT. Early diagnosis and surgical or radiological intervention is the corner stone to save the recipient. Despite the frequent size discrepancy, Splenic artery could be a reasonable alternative for graft arterial revascularization in LDLT when inadequate recipient HA flow is present. This approach is particularly suitable when splenomegaly is present. Treatment of late arterial complications is mainly non surgical, angiography and stenting is successful in late HAS, medical treatment (anticoagulation) is successful in late HAT.

\section{REFERENCES}

1. Guba M, Adcock L, MacLeod C, Cattral M, Greig P, et al. (2010): Intraoperative 'no go' donor hepatectomies in living donor liver transplantation. Am J Transplant 10: 612618.

2. Lee SG, Hwang S, Kim KH, Ahn CS, Moon DB, et al. (2009): Toward 300 liver transplants a year. SurgToday 39: 367-373.
3. Freise CE, Gillespie BW, Koffron AJ, Lok AS, Pruett TL, et al. (2008): Recipient morbidity after living and deceased donor liver transplantation: findings from the A2ALL Retrospective Cohort Study. Am J Transplant 8: 2569-2579.

4. Eguchi S, Takatsuki M, Hidaka M, Tajima Y, Kanematsu T (2008): Evolution of living donor liver transplantation over 10 years: experience of a single center. Surg Today 38: 795-800.

5. Olthoff KM, Merion RM, Ghobrial RM, et al. (2009): Outcomes of 385 adult-to-adult living donor liver transplant recipients: A report from the A2ALL consortium. Ann Surg.; 242: 314-325.

6. Khalaf H (2010): Vascular complications after deceased and living donor liver transplantation: a single-center experience. Transplant Proc 42: 865-870.

7. Steinbrück $K$, Enne $M$, Fernandes $R$, Martinho JM, Balbi E, Agoglia L, Roma J, Pacheco-Moreira LF (2011): Vascular complications after living donor liver transplantation: a Brazilian, single-center experience. Transplant Proc. 43(1):196-8.

8. Gunsar F, Ronaldo N, pastacaldi S, et al. (2003): Late hepatic artery thrombosis after orthotopic liver transplantation. Liver transpl. 9:605-611.

9. Henrik P and Ronald B (2009): Evolving Surgical Approaches in Liver Transplantation. Seminars in Liver Disease. Liver Transplantation. 29(1):121-133.

10. Polak W, Peeters P, Slooff M (2009): The evolution of surgical techniques in clinical liver transplantation. A review, Clin Transplant DOI: 10.1111/j.1399-0012. 2009.

11. Takatsuki M, Chiang YC, Lin TS, Wang CC, Concejero A, Lin CC, Huang TL, Cheng YF, Chen CL (2006): Anatomical and technical aspects of hepatic artery reconstruction in living donor liver transplantation. 140 (5): 824- 828 . 
12. Panossian A, Diamond I, Fecteau A, Grant D, Zuker R (2009): Hepatic artery microvascular anastomosis in pediatric living donor liver transplantation: a review of 35 consecutive cases by a single microvascular surgeon. J Reconstr Microsurg. 25 (7): 439-443.

13. Lerner SM, Markmann J, Jurim $\mathrm{O}$, et al. (2005): Retransplantation. In: Busuttil RW, Klintmalm GB, eds. Transplantation of the Liver, 2nd ed. Philadelphia: WB Saunders. 767.

14. Bekker J, Ploem S, de Jong KP (2009): Early hepatic artery thrombosis after liver transplantation: a systematic review of the incidence, outcome and risk factors. Am J Transplant. 9:746.
15. Marshall MM, Muiesan $P$, Srinivasan $P$, Kane PA, Rela M, Heaton ND, Karani JB, Sidhu PS (2001): Hepatic artery pseudoaneurysms following liver transplantation: incidence, presenting features and management. Clinical radiology. 56 (7): 579-87.

16. Maleux G, Pirenne J, Aerts R, Nevens F (2005): Hepatic artery pseudoaneurysm after liver transplantation: definitive treatment with a stent-graft after failed coil embolisation. The British journal of radiology. 78 (929): 453-6. 


\title{
Khaled Zaky Mansour, et al.,
}

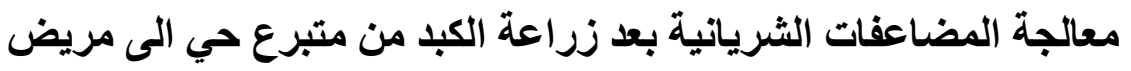 \\ خالد زكى منصور، محمد محمد بهاء الدين، أحمد حسين عبد الحافظ، كمال ممدوح كمال، \\ محمد احمد فتحى البهنسى \\ قسم الجر احة العامة، كلية الطب- جامعة عين شمس لمس
}

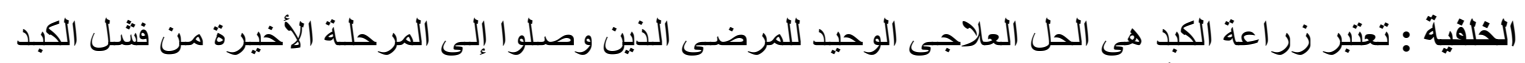

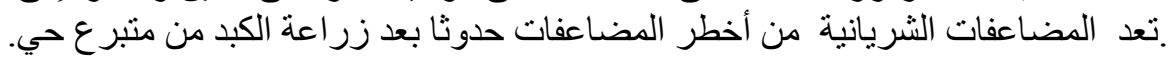

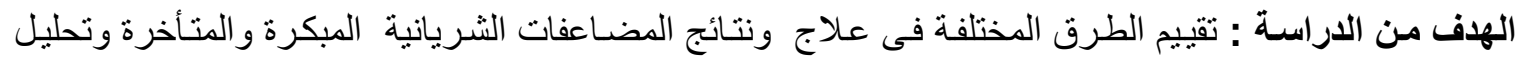

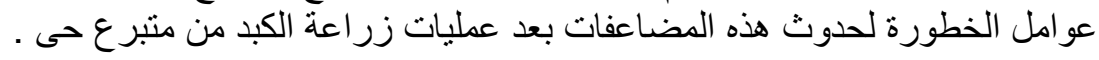

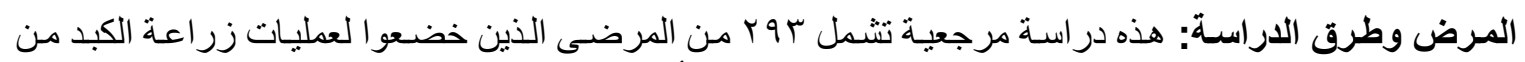

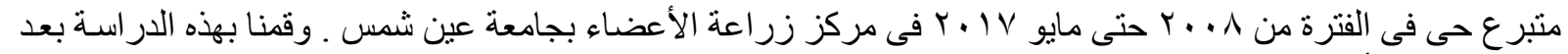

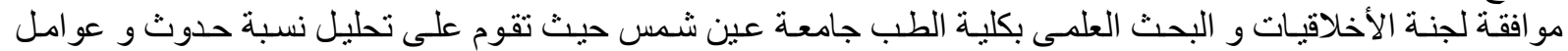

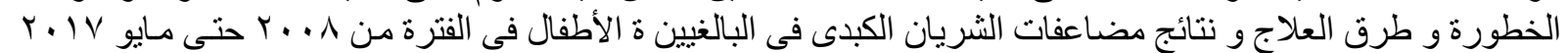

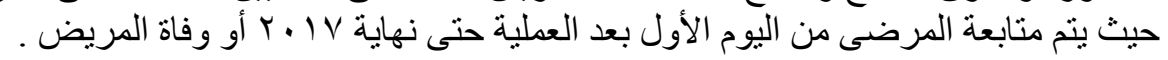

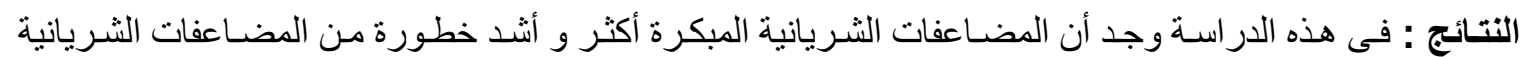

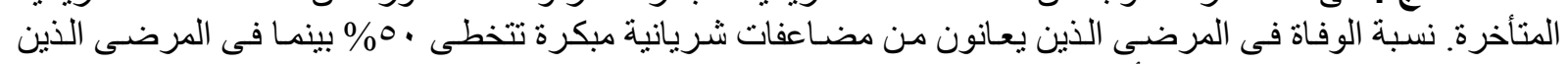

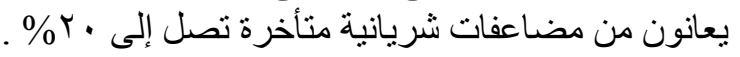

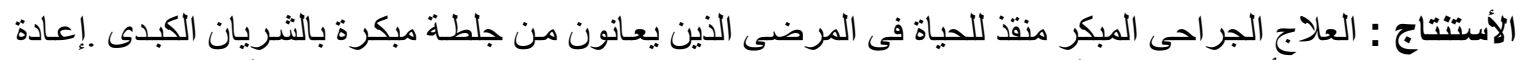

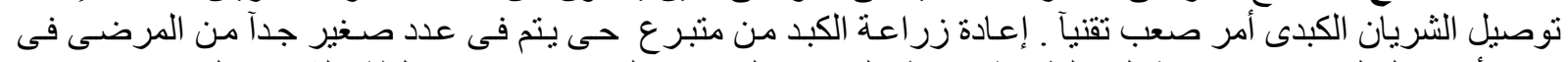

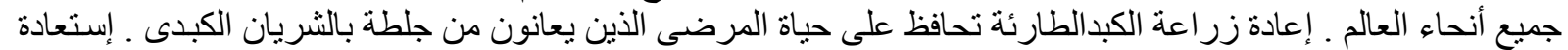

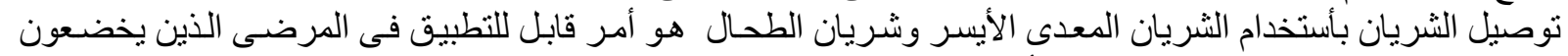

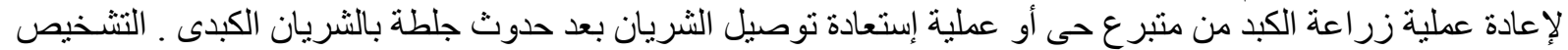

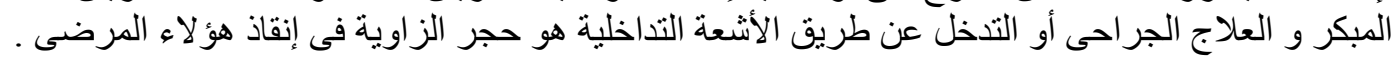

